\title{
Caracterización de fincas productoras del cultivo de palto en la región Moquegua, Perú
}

\author{
Characterization of farms producing avocado \\ cultivation in the Moquegua region, Peru
}

Edgar Bedoya Justo ${ }^{*}$, Alberto Julca Otiniano ${ }^{1}$

\section{RESUMEN}

El objetivo de este trabajo fue caracterizar las fincas productoras de palto en la región Moquegua. Se trabajó con una población de 428 productores de la cual se tomó una muestra irrestricta aleatoria $(n=92)$ para realizar una encuesta que incluía preguntas relacionadas con aspectos técnicos y socioeconómicos de las fincas. Los resultados indican una débil organización, predominio de minifundio, con bajos rendimientos y muy baja inversión. Hay una tendencia al cambio del sistema de riego tecnificado, con apoyo del gobierno local. Se encontró que la mayor parte posee policultivos y destaca el cultivo de alfalfa, orientado a la crianza de ganado vacuno, lo cual permite que se generen más ingresos económicos para la familia. En cuanto a cultivares, se usan principalmente Hass y Fuerte. Los mecanismos de comercialización son variados y se carece de un sistema de comercialización. Asimismo, faltan mecanismos de organización para negociar con los compradores, ya que la mayoría exporta a través de intermediarios o acopiadores y en la finca. El análisis de conglomerado reúne a las fincas en cinco grupos. Uno de los grupos estuvo conformado por 14 fincas $(15 \%)$, que usan un solo cultivar, invierten más y obtienen los más altos rendimientos.

Palabras clave: aguacate, diagnóstico agrícola, Perú, Persea americana.

\begin{abstract}
The objective of the present work was to characterize the farms producing avocado cultivation in the Moquegua Region. We worked with a population of 428 producers from which an unrestricted random sample was taken $(n=92)$ to carry out a survey that included questions related to the farms' technical and socio-economic aspects. The results indicate a weak organization, predominance of smallholdings, with low yields and little investment; there is a tendency to change the technical irrigation system, with support from the local government. It was found that most of them have polycultures, highlighting the cultivation of alfalfa, aimed at raising cattle, which allows for a little more income for the family. As for cultivars, Hass and Fuerte are mainly cultivated; Marketing mechanisms are varied; there is no marketing system; likewise, there is a lack of organizational mechanisms to negotiate with buyers, as most export through intermediaries or gatherers and on the farm. Cluster analysis groups farms into five groups; one groups consisted of 14 farms; these cultivated a cultivar, invested more, and obtained the highest yields.
\end{abstract}

Keywords: avocado, agricultural diagnosis, Peru, Persea americana.

\section{Introducción}

El palto (Persea americana Mill.), conocido también como aguacate, se originó en México, de donde se extendió a la América Central y a las Antillas, y a la parte norte de la América del Sur. Según Garcilaso de la Vega, el Inca, esta especie frutal fue introducida en el Perú al valle del río Urubamba en 1540 por su bisabuelo Túpac Yupanqui, luego de conquistar a la tribu Palta, en el norte de Tumbes, Perú (Franciosi, 1992 y Ferreyra et al., 2005). La superficie mundial cosechada de palto ha crecido de 339495 ha en el año 2000 a 486040 ha en el año 2014 (Ministerio de Agricultura y Riego -Minagri- 2015). En el año 2017 la producción mundial alcanzó los 6048 5083 t, y México fue el principal productor con 2029886 t. El Perú se ubica en el puesto tres con 466758 t y es el segundo país exportador con 247 mil toneladas (12,4\% del total) después de México, que representa el 45\% del total exportado (Minagri, 2019). En nuestro país se tiene un total de 25751 ha

\footnotetext{
$1 \quad$ Universidad Nacional Agraria La Molina (UNALM), Lima, Perú

* Autor por correspondencia: edgbedoya@gmail.com
} 
de palto (Minagri, 2015) y los principales cultivares son Fuerte, Hass, Zutano, Ettinger, Naval y Criolla (Buendía, 2015). Las regiones de La Libertad, Lima, Ica, Junín y Ancash representan el 77\% de la producción total, con un rendimiento promedio de $12.030 \mathrm{~kg} / \mathrm{ha}$. Moquegua se sitúa en sexto lugar en términos de área cultivada. En esta región es el principal cultivo frutícola con una superficie cultivada de 963 ha y un rendimiento promedio de $5.255 \mathrm{~kg} / \mathrm{ha}$ (Gobierno Regional Moquegua, 2018). La mayor parte de las plantaciones son del cultivar Hass y en menor proporción Fuerte, Nabal, entre otras. El cultivar exportable es Fuerte y en menor proporción Hass. Entre los principales problemas sanitarios en la región destacan la "pudrición radicular" (Phytophthora cinnamoni) que disminuye la productividad (Huaman et al., 2015) y la "muerte regresiva" (Lasiodiplodia theobromae). Según el Gobierno Regional de Moquegua (2019), el bajo nivel tecnológico de los productores de palto no permite realizar adecuadamente las labores culturales como manejo de suelos, riegos, manejo de malezas, abonamiento, podas y enfermedades. A pesar de todos estos problemas, en los 10 últimos años, la superficie cultivada se ha ido incrementando progresivamente de 370 ha a 963 ha. Sin embargo, para una mejor planificación y distribución más eficiente de los recursos destinados a mejorar el funcionamiento de los sistemas productivos, es necesario hacer una caracterización de las fincas, como paso previo para cualquier proyecto posterior. El agrupamiento de las explotaciones busca maximizar la homogeneidad dentro de los grupos y la heterogeneidad entre estos (Santistevan et al., 2015 y Márquez, 2016). Bolaños (1999) señala que la caracterización no es más que la descripción de las principales características y las múltiples interrelaciones en las organizaciones. Para Malagon y Praguer (2001), la caracterización es una etapa determinante para desarrollar el método de investigación en sistemas de producción y consiste en establecer un conjunto de variables que distinguen a una zona o unidad de producción en particular y que la hace diferente a otras. También Balda (2012), citado por Santisteban et al. (2015), indica que la caracterización no es más que la descripción de las principales características y las múltiples interrelaciones entre las organizaciones, que sirven también para definir la línea de base y establecer relaciones entre variables sociales, económicas, ambientales y productivas en un sistema. Por lo expuesto, este trabajo se realizó con el objetivo de caracterizar las fincas productoras del cultivo de palto en la región Moquegua, Perú.

\section{Materiales y métodos}

El cultivo de palto en el departamento de Moquegua se ubica en las provincias de Mariscal Nieto y General Sánchez Cerro, que concentran el total del área cultivada (963 ha), distribuidas entre los 1400 y $2500 \mathrm{msnm}$ y pertenecientes a 758 productores (Gobierno Regional de Moquegua, 2019). El trabajo de investigación se realizó en la provincia Mariscal Nieto (Figura 1), ubicada
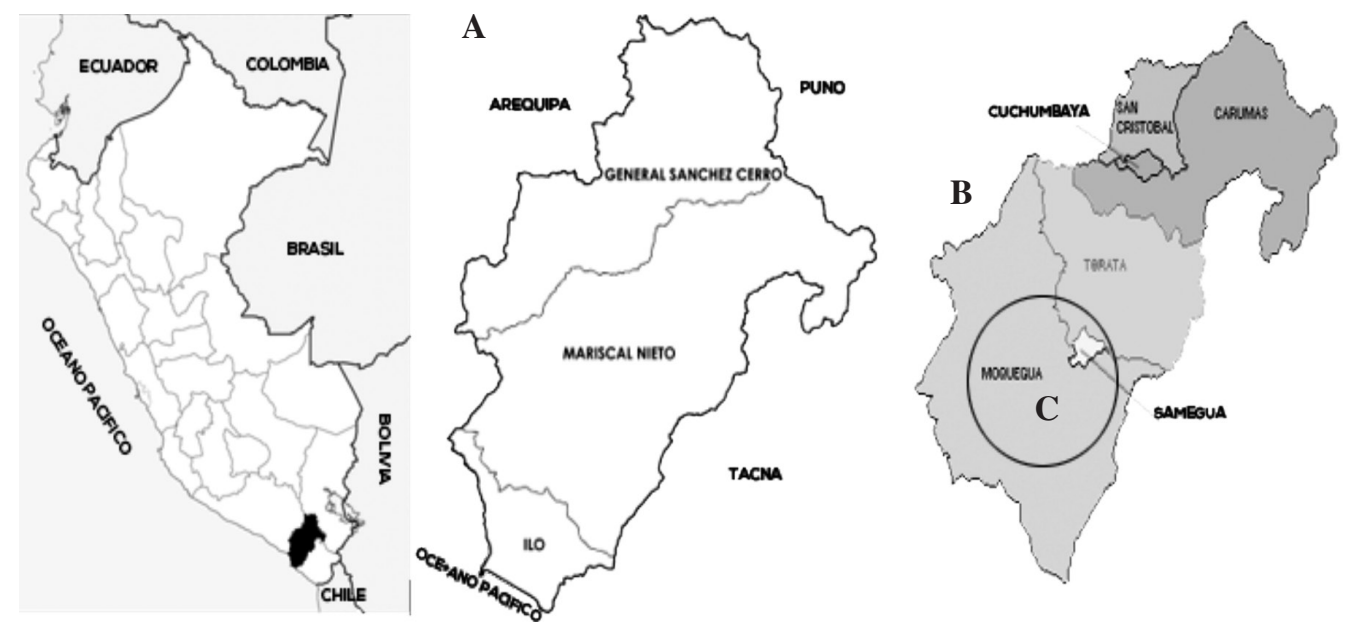

Figura 1. Mapa del Perú (A), Región de Moquegua (B) con sus tres provincias y la Provincia Mariscal Nieto (C), donde la zona de estudio está marcada con un círculo. 
entre las coordenadas $15^{\circ} 57^{\prime}$ a $17^{\circ} 53^{\prime}$ de LS y $70^{\circ} 00$ a $71^{\circ} 23^{\prime}$ de LO y una extensión territorial de $7.369,19 \mathrm{~km}^{2}$. Cuenta con seis distritos, tres de los cuales son productores de palta (Moquegua, Samegua y Torata). La temperatura máxima es de $27,10^{\circ} \mathrm{C}$, la mínima de $8,90^{\circ} \mathrm{C}$ (Servicio Nacional de Meteorología e Hidrología -Senamhi-2017) y con escasa precipitación pluvial. Entre estos distritos se tiene un total de 463 ha, con 428 productores (Gobierno Regional de Moquegua, 2017). De esta población se obtuvo una muestra irrestricta aleatoria [ $\mathrm{n}=92]$ (Sheaffer et al. 1987). La información de cada una de las fincas se logró a través de encuestas que incluyeron un conjunto de preguntas relacionadas con aspectos técnicos y socioeconómicos. La encuesta fue procesada en una hoja de cálculo. Para determinar el agrupamiento de fincas se realizó un análisis de conglomerados (cluster analysis) por el Método de Ward con una distancia euclidiana cuadrada de 900 (Andrade, 1985, citado por Tuesta et al., 2014).

\section{Resultados y discusión}

\section{Características del productor}

La Figura 2 muestra que las personas responsables de la finca son mayormente del sexo masculino
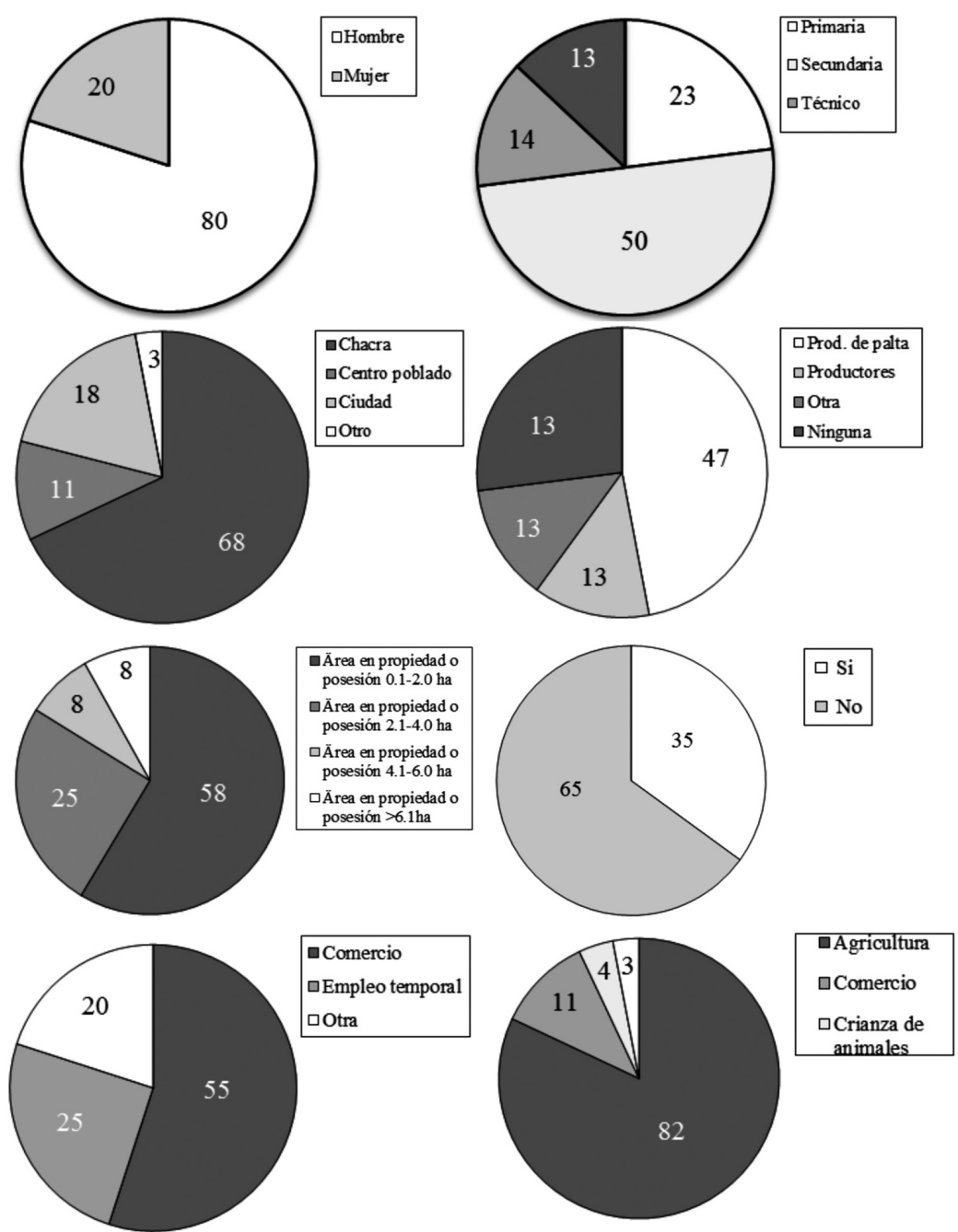

Figura 2. Sexo, lugar de residencia, tamaño de la finca y otra actividad económica (lado izquierdo). Nivel de instrucción, tipo de organización, actividad económica y origen de ingresos mensuales de los productores de palta (lado derecho). Los datos están en porcentajes. 
(80\%), con edades comprendidas entre los $40 \mathrm{y}$ 60 años (62\%). Les sigue un grupo cuyas edades están entre los 61 y 70 años (25\%) y finalmente los agricultores que tienen entre 71 y 80 años (13\%). El nivel de instrucción de los productores es muy variado: la mitad (50\%) tiene educación secundaria, seguido de educación primaria (23\%) y hay un grupo importante con estudios superiores que van desde el nivel técnico hasta el universitario (27\%). En cuanto a las personas que viven en el hogar, hay un predominio (53\%) de dos integrantes, mayormente los esposos; con uno (20\%); con tres (17\%), siendo el tercero o más integrantes menores de edad o personal de apoyo en las labores agrícolas; con cuatro integrantes (13\%), y más de cuatro (3\%).

Con respecto al lugar de residencia, es mayormente en la finca (68\%). Un segundo grupo $(18 \%)$ reside en la ciudad, un tercer grupo en centros poblados $(11 \%)$ y un pequeño grupo $(3 \%)$ en otros lugares. El tipo de organización al cual pertenecen los productores de palta es diverso. Destaca en primer lugar la organización de palteros (47\%); luego se tiene (27\%) de productores que no pertenecen a ninguna organización o no están organizados; en tercer lugar (13\%) pertenecen a la organización de productores múltiples, y finalmente $(13 \%)$ pertenecen a otras organizaciones. En lo referente al tamaño de la posesión de las tierras agrícolas, mayormente (58\%) tienen entre 0,1 y 2,0 ha; en segundo lugar (25\%) entre 2,1 y 4,0 ha; un tercer grupo (8\%) y un reducido grupo (8\%) entre 4,1 y 6,0 ha. Según el Censo Nacional Agrario (CENAGRO) 2012, entre el año 1994 y el 2012 hubo un incremento del tamaño de la unidad agropecuaria en Moquegua de 0,1 a 5,0 ha, incrementándose del 81,6 al 91,2\%, respectivamente. También hay un predominio de la actividad económica, donde (65\%) se dedican exclusivamente a la actividad agropecuaria y (35\%) tienen además de la actividad agropecuaria, otra actividad económica. Dentro de las otras actividades económicas a las que se dedican los productores, mayormente predomina el comercio (55\%). Luego (20\%) tienen empleos eventuales y en tercer lugar (25\%) realizan otras actividades. Los ingresos mensuales que perciben los productores son principalmente de la agricultura (82\%); en segundo lugar se tiene la actividad comercial (11\%), seguidamente la crianza de animales $(4 \%)$ y un $(3 \%)$ otras actividades.

Con relación a capacitación, un $61 \%$ indicó que sí recibió y provino del Minagri (30\%), seguido del municipio provincial (29\%), del Gobierno Regional (17\%), Organismos Nacionales No Gubernamentales (ONG), los propios productores y otros (24\%). Un $39 \%$ de los productores mencionó que no recibió ninguna capacitación. Los temas en los que recibieron capacitación fueron mayormente el manejo de plagas y enfermedades (34\%), en segundo lugar (24\%) la fertilización, en tercer lugar (18\%) el comercio, en cuarto lugar (13\%) la cosecha y poscosecha, y finalmente (11\%) abonos orgánicos, almácigos y otros. E lo referente a los temas en que los agricultores desearían ser capacitados, coinciden en promedio con los de capacitación recibidos.

\section{Características de la finca}

Según se muestra en la Figura 3, en la provincia Mariscal Nieto, la variedad de palto que se cultiva mayormente es la Hass (47\%). Le sigue la Fuerte $(35 \%)$ y hay un grupo de productores (16\%) que cultivan ambas variedades. En cuanto a los portainjertos (patrones), la variedad Zutano (56\%) es la más utilizada, seguida de Topa Topa (35\%), Duke 7 y la Mexicana (2\%). La edad de las plantaciones fluctúa mayormente entre 4,0 y 8,0 años (65\%), seguida de las mayores a 16 años (18\%), otro grupo entre 12,1 y 16,0 años $(12 \%)$ y finalmente las que tienen entre 8,1 y 12,0 años $(5 \%)$. La densidad de plantación ( $\mathrm{N}^{\circ}$ plantas/ha) es variable. La mayor parte usa entre 401 y 500 plantas/ha (35\%), otro grupo tiene entre 200 y 300 plantas/ha (30\%), seguido de los que utilizan más de 500 plantas/ha (25\%) y los que poseen entre 301 y 400 plantas/ha (12\%).

En cuanto al manejo del agua de riego, el 52\% de los productores de palto riega por gravedad, un 43\% utiliza riego tecnificado y solo un pequeño grupo (3\%) usa ambos sistemas de riego. Según el CENAGRO (2012), en Moquegua el 94,9\% de productores utiliza riego a gravedad y solo un 5,1\% riego tecnificado, predominando mayormente el riego por goteo. Dentro de los otros cultivos destaca la alfalfa (75\%), seguida de las hortalizas (10\%), la vid (8\%) y otros cultivos (7\%). Según el Gobierno Regional de Moquegua (2018), el 67,2\% del área cosechada en el departamento de Moquegua es del cultivo de alfalfa. La semilla o plantones que utilizan (61\%) se trae de otros lugares, aunque también de la misma zona (30\%), y hay un pequeño grupo que usa su propia semilla o plantones $(9 \%)$. Del 

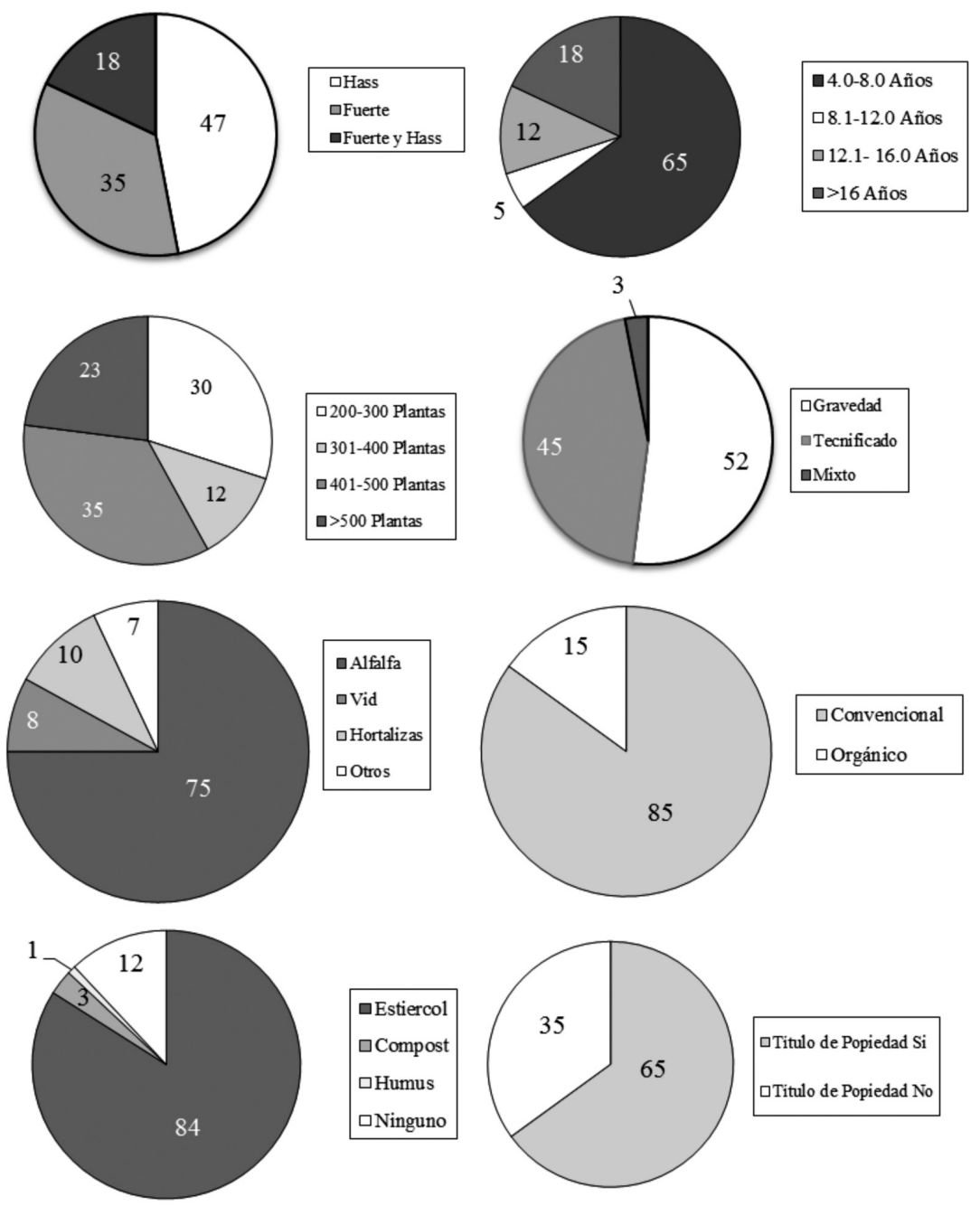

Figura 3. Variedad de palto, densidad de plantación, otros cultivos y tipo de insumo orgánico (lado izquierdo). Edad de plantaciones, manejo de agua, tipo de agricultura y propiedad de la tierra de los productores de palta (lado derecho). Los datos están en porcentajes.

material de siembra que se utiliza para el desarrollo de sus cultivos, el $27 \%$ son plantones injertados certificados, el $17 \%$ plantones injertados y el $9 \%$ plantones (patrones) certificados o comunes. En cuanto a la semilla, el $29 \%$ usa semilla certificada y el $9 \%$ semilla común.

En lo referente al tipo de agricultura que desarrollan los productores, es mayormente convencional (85\%) y solo $15 \%$ del tipo orgánico o ecológico. Entre los insumos de tipo orgánico que aplican destaca el estiércol con un $84 \%$, un $12 \%$ que no aplica ningún insumo orgánico y solo un $4 \%$ que utiliza compost y humus. El CENAGRO (2012) indica que en el departamento de Moquegua el 79,9\% de productores aplica guano, estiércol u otro abono orgánico y un $20,1 \%$ no aplica. Con relación a la propiedad de la tierra, el $65 \%$ tiene título de propiedad debidamente registrado en la Superintendencia Nacional de los Registros Públicos (SUNARP) y el 35\% no tiene título de propiedad. En cuanto a servicios básicos de escuela y posta médica, la mayoría (65\%) no cuenta con ningún servicio en la zona, el $17 \%$ tiene servicio de escuela pública, el $7 \%$ dispone de posta médica y el $11 \%$ de escuela y posta médica. Asimismo, el 65\% tiene servicio de agua potable y luz, el $10 \%$ solo cuenta con luz, el $9 \%$ con agua potable y $16 \%$ no dispone de agua potable ni luz. Con respecto a vías 
de comunicación, todos tienen vías y servicios de acceso hacia la ciudad, el $91 \%$ cuenta con telefonía y tan sólo el $9 \%$ no posee telefonía.

\section{Características de la producción}

Según la Figura 4, el rendimiento promedio del cultivo de palto está mayormente entre 6,1 y 8,0 t/ha (60\%), un segundo grupo produce entre 8 y 10 t/ha (20\%), un tercer grupo produce más de 10 t/ha (15\%) y el grupo más pequeño produce entre 4,0 y 6,0 bt/ha (5\%). De acuerdo con el Gobierno Regional Moquegua (2018), el departamento de Moquegua tiene un rendimiento promedio de palta de $5255 \mathrm{~kg} / \mathrm{ha}$. El precio más alto $(\mathrm{S} / 6,1 \mathrm{a} 7,0 / \mathrm{kg})$ lo alcanza un grupo de productores (15\%), mientras que en el otro extremo está el $8 \%$ que vendió entre $\mathrm{S} / 3,0$ y $4,0 / \mathrm{kg}$, seguido por los que vendieron $\mathrm{S} / 5,1$ a $6,0 / \mathrm{kg}(30 \%)$ y S/4,1 a 5,0/kg. Según el Gobierno Regional Moquegua (2018), el precio promedio al consumidor de la palta Fuerte fue de S/9,02/kg. La calidad del fruto para su comercialización es por el tamaño (58\%), por el color (20\%), calibre (18\%) y por la forma (4\%). Los lugares donde se realiza la comercialización son variados, no existe un sistema de comercialización establecido y faltan mecanismos de organización para negociar con los compradores. La mayor parte (53\%) lo exporta a través de intermediarios o acopiadores y en la finca, un grupo lo vende al mercado local (30\%), el $14 \%$ lo comercializa directamente en las ferias y un reducido grupo (3\%) bajo otras modalidades.

La inversión anual de los productores en el cultivo del palto es muy variada. El $4 \%$ invierte más de S/4.000/ha, mientras que el 25\% invierte $\mathrm{S} / 1.000$ a $2.000 /$ ha, un $35 \%$ invierte $\mathrm{S} / 2.001$ a $3.000 /$ ha y el $30 \%$ invierte $\mathrm{S} / 3.001$ a $4.000 /$ ha. La inversión es orientada mayormente al manejo del riego, malezas y cosecha. El ingreso mensual de los productores en Moquegua está mayormente
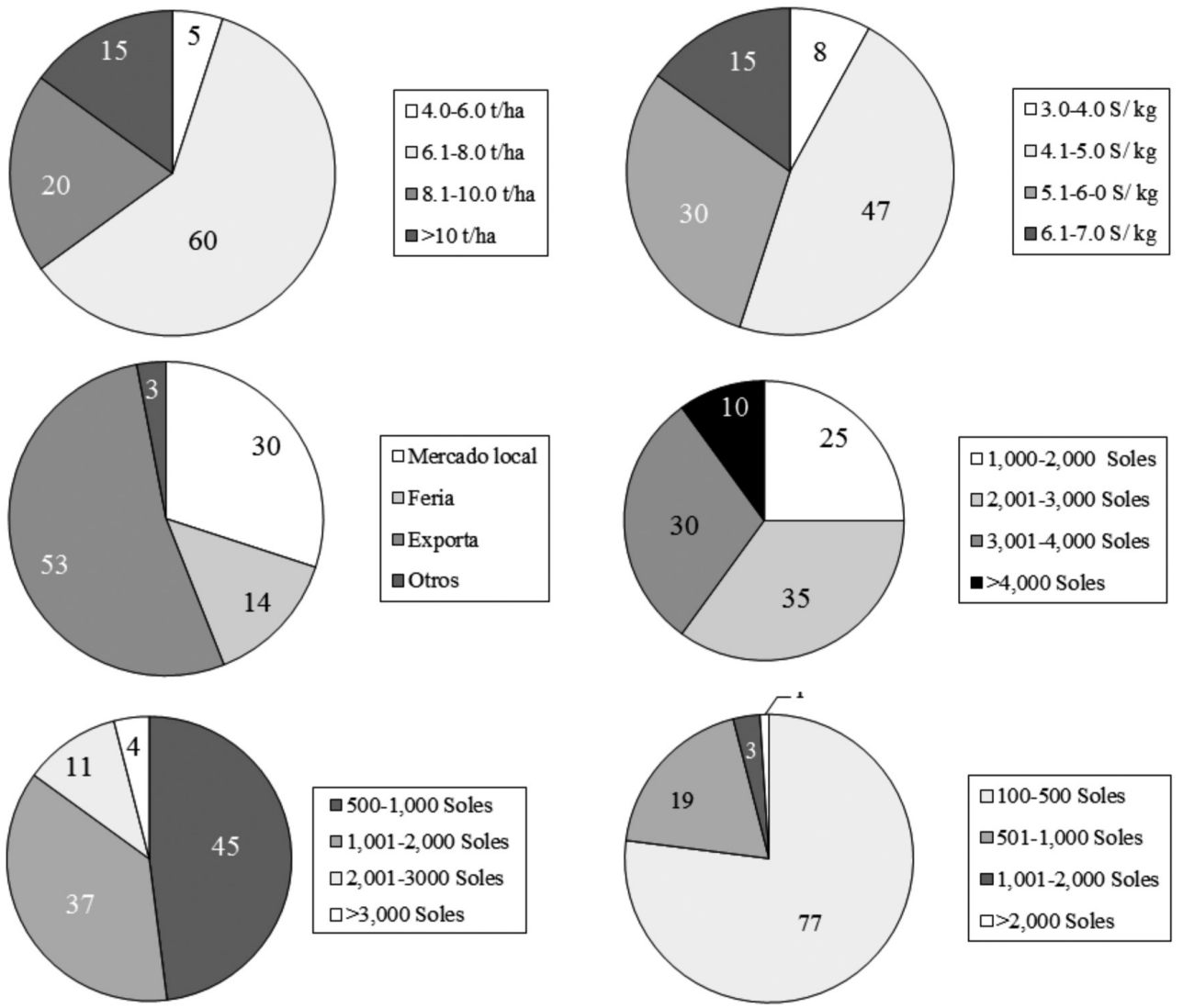

Figura 4. Rendimiento, lugar de comercialización e ingreso mensual (lado izquierdo). Precio de la palta, inversión anual y otros ingresos de los productores de palta (lado derecho). Los datos están en porcentajes. 
entre los S/500 y 1.000 (45\%); el segundo grupo es de $S / 1.001$ a 2.000 (37\%), le siguen aquellos que tienen ingresos de $\mathrm{S} / 2.001$ a $3.000(11 \%)$ y hay un grupo (4\%) con ingresos mayores a S/3.000. El ingreso mensual por crianza de animales es mayoritariamente bajo (77\%) entre $\mathrm{S} / 100$ y 500; en segundo lugar hay un grupo (19\%) que tiene ingresos entre $\mathrm{S} / 501$ y 1.000 , otro grupo minoritario (3\%) con ingresos entre S/1.001 y 2.000 y finalmente un grupo muy pequeño (1\%) con ingresos mayores a 2.000 soles. La crianza de animales está destinada mayormente a la venta en el mercado local (50\%), para autoconsumo (37\%), la comercialización en ferias (12\%) y otros (1\%). Según el Minagri (2015), en el Perú más del 40\% del Valor Bruto de la Producción (VBP) no tiene al mercado como destino, ya que un porcentaje muy alto es dirigido al autoconsumo, sobre todo en las pequeñas chacras del interior del país. Asimismo, el Gobierno Regional Moquegua (2018) reporta que la crianza de animales está orientada mayormente a vacunos, cuyes, aves, ovinos y porcinos. Los ingresos en su mayoría provienen de la producción de leche, ya que en promedio se tienen 29.594 unidades y bajo ordeño 5.295 unidades, con una producción de leche de $7,93 \mathrm{~kg} /$ día a un precio promedio de $\mathrm{S} / 1,49 / \mathrm{kg}$.

El análisis de conglomerado por el Método de Ward y con una distancia euclidiana cuadrada de 900 reúne a las fincas en cinco grupos. Uno de los grupos estuvo conformado por 14 fincas (de rojo de la Figura 5), las cuales se dedican al mismo cultivar, invierten más y obtienen los más altos rendimientos. El agrupamiento de fincas es importante porque las acciones futuras podrían realizarse para cada grupo y ya no de manera individualizada (Criollo et al., 2016, citados por Anzules et al., 2018), dado que los grupos se forman por similitud entre los elementos que los componen y se podría asumir que aquellas fincas que se agrupan solo presentan diferencias significativas con el resto (Castro et al., 2012, citados por Anzules et al., 2018).

En general, los resultados son parecidos o similares a los reportados por los organismos oficiales del estado. El Minagri (2002) y el CENAGRO (2012) señalan que el productor en la costa peruana pertenece mayormente al sexo masculino y la mayoría tiene solamente educación primaria y secundaria. A pesar de los notables avances en términos de educación en el país, los niveles en el sector rural aún se mantienen muy por debajo del resto de la población. Ello genera poca capacidad de los productores para la innovación tecnológica. También se indica que hay migración de los jóvenes hacia las ciudades, y solo quedan en el campo los padres y menores. Incluso hay campos de cultivo que han sido abandonados. Muchos productores, por motivos de falta de mano de obra y seguridad en sus fincas, tienen su residencia en el campo. Asimismo, el Minagri (2002) señala que en el año 1994, solo el 35\% de los productores agropecuarios declararon pertenecer a alguna organización, mientras que en este estudio se reporta que el $47 \%$ de productores de palto están organizados. El capital institucional de los productores organizados es importante para ordenar y organizar la producción, y para

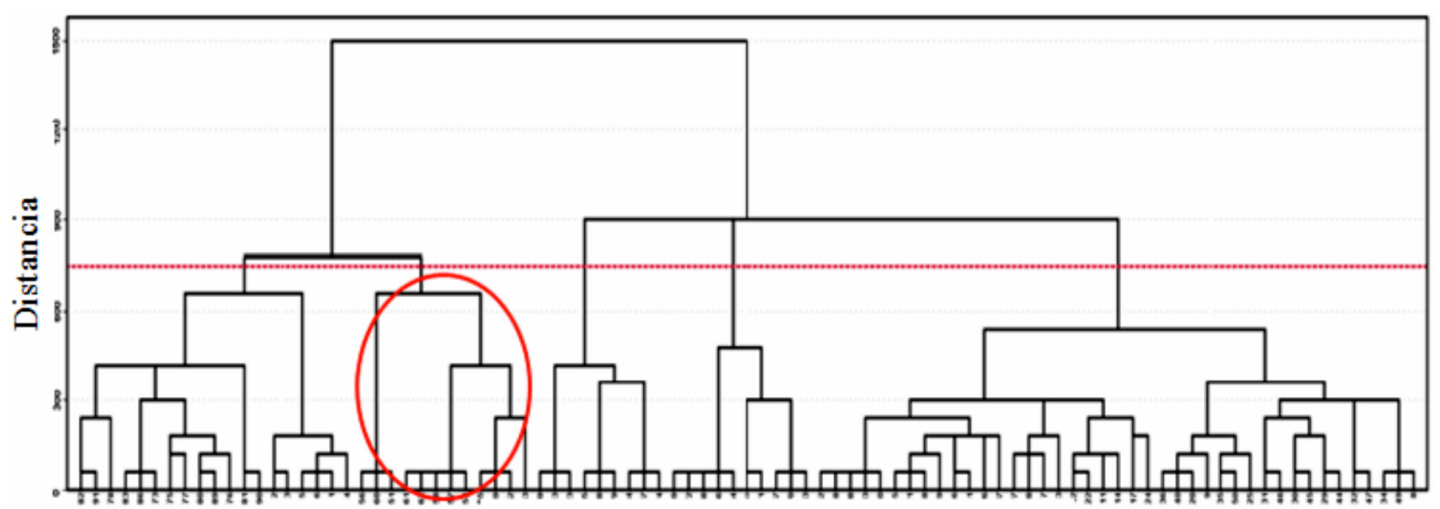

Fincas productoras de palta

Figura 5. Agrupamiento de fincas productoras de palta en la provincia Mariscal Nieto (Moquegua, Perú) con el Método de Ward y una distancia Euclidiana Cuadrada. 
evitar consecuencias indeseables, tales como la sobreproducción y caída de precios (Minagri, 2019). La asociatividad constituye una oportunidad para el desarrollo de la agricultura, ya que los productores pueden organizarse para mejorar tanto la producción como la comercialización del producto y conseguir precios bajos para la compra de los insumos y agroquímicos (Molero et al., 2007, citados por Cáceres y Julca, 2018). Hay una marcada reducción del tamaño de las unidades agropecuarias y dispersión de las parcelas, lo cual se convierte en un gran obstáculo para la rentabilidad. Según el CENAGRO (1994), más del $70 \%$ de las unidades agropecuarias cuenta con una extensión menor a cinco hectáreas y ocupa menos del $6 \%$ del total de la superficie agrícola nacional, y de acuerdo al CENAGRO (2012) hubo un incremento en la variación porcentual del 26,9\%. La reducida extensión de las parcelas genera obstáculos en los cultivos de exportación, por lo que como una alternativa para obtener mayores ingresos, los productores dedican parte de su tiempo a otras actividades económicas, como la crianza de animales, el comercio y empleo temporal en los municipios. Proaño y Lacroix (2014), citados por Anzules et al. (2018), indican que la agricultura, a pesar de ser la actividad principal, se complementa con otras actividades económicas como cría de ganado, artesanías, caza y pesca, y también con trabajos fuera de la finca, a tiempo parcial, estacional e intermitente, que les permiten obtener otros ingresos y atenuar situaciones críticas en condiciones adversas (Bayona y Muñoz, 2009, citados por Anzules et al., 2018). La diversificación de las fincas ha sido, para la mayoría de los pequeños productores, la opción que les permite lograr otros ingresos que ayuden a mejorar su economía y atenuar las situaciones críticas que por épocas atraviesan algunas actividades productivas (Vargas-Jarquín y Sánchez-Benavides, 2015, citados por Aquino et al., 2108).

En los últimos años ha habido una tendencia a plantar el cultivar Hass, en reemplazo de Fuerte, debido a las expectativas por exportar y con densidades mayores a 500 plantas/ha, con riego presurizado (goteo). Sin embargo, el manejo es convencional y con poca inversión, lo que trae como consecuencia una baja productividad y rentabilidad. Según Vélez (2015), si el productor no es económicamente sostenible, la industria en su conjunto está amenazada. Asimismo, el bajo nivel tecnológico de los productores no permite realizar adecuadamente las labores culturales como manejo de los suelos, riegos, abonamiento y podas. A ello se suma el desconocimiento de las medidas sanitarias para el manejo de enfermedades.

En los últimos diecisiete años (2001-2018) ha habido en el Perú un notable crecimiento, de manera que en 2018 se registró un nivel récord de exportación del cultivar Hass, en valor y volumen, constituyéndose por primera vez en el segundo producto más importante de agroexportación, con US\$ 717 millones de dólares (Minagri, 2019). Sin embargo, en Moquegua el cultivo que más destaca en el área es la alfalfa. A pesar de los bajos rendimientos, se exporta palta al vecino país de Chile. Según el Gobierno Regional de Moquegua (2018), en el año 2017 se exportaron 263,83 t de los cultivares Fuerte y Hass, en la misma proporción. La comercialización es a través de intermediarios, donde esta falta de relación con el mercado es causa y consecuencia del bajo poder de negociación con que cuentan los productores (Minagri, 2019).

\section{Conclusión}

De lo estudiado, se concluye que predomina el minifundio con una baja productividad y rentabilidad, a pesar de haber un cambio por instalar un nuevo cultivar (Hass), con alta densidad y riego tecnificado. Sin embargo, el manejo es convencional, con baja inversión, lo cual tiene como causa principal el deficiente manejo técnico del cultivo. Una de las principales características de la mayoría de los productores es que poseen huertos tipo vergel, donde cultivan mayormente alfalfa, hortalizas y otros frutales como el palto, que es el principal cultivo permanente en la región de Moquegua. También se dedican a la crianza de animales. Por otra parte, existe un débil vínculo entre el productor y el consumidor final. 


\section{Literatura Citada}

Anzules, V.; Borjas, R.; Castro, V.; Julca, A.

2018. Caracterización de fincas productoras de cacao

(Theobroma cacao L.) en Santo Domingo de Los Tsáchilas, Ecuador. Bosques Latitud Cero, 8(2): 39-50.

Aquino, V.; Camarena, F.; Julca, A.; Jiménez, J.

2018. Caracterización multivariada de fincas productoras de tarwi (Lupinus mutabilis Sweet) del Valle de Mantaro, Perú. Sciencia Agropecuaria, 9(2): 269-279.

Bolaños, O.

1999. Caracterización y tipificación de organizaciones de productores y productoras. Unidad de Planificación Estratégica. Ministerio de Agricultura y Ganadería. XI Congreso Nacional Agronómico / I Congreso Nacional de Extensión. Costa Rica.

Buendía, $\mathrm{M}$.

2015. Cultivo, producción y comercialización de paltos. Editorial MACRO. Lima, Perú. 216 p.

Cáceres, H.; Julca, A.

2018. Caracterización y tipología de fincas productoras de vid para pisco en la región Ica-Perú. IDESIA (Chile), 36(3): 35-43.

Ferreyra, R.; Pilar, M.; Maldonado, P.; Toledo, C.; Barrera,

C.; Celedón, J.

2005. El cultivo del palto. Boletín INIA $N^{o} 129$. Instituto de Investigaciones Agropecuarias. Chile. $80 \mathrm{p}$.

Franciosi, R.

1992. El cultivo de palto en el Perú. FUNDEAGRO. Lima, Perú. 89 p.

Gobierno Regional de Moquegua.

2018. Estadística Agraria 2017. Moquegua, Perú: Gerencia Regional de Agricultura. Disponible en: https://www. agromoquegua.gob.pe/doc/anuarios/ANUARIO_ ESTADÍSTICO_AGROPECUARIO_2017_OK.pdf. Consultado: 10/may/2019.

Gobierno Regional Moquegua.

2019. Estadística agraria 2018. Moquegua: Gerencia Regional de Agricultura. Moquegua, Perú. 179 p.

Huamán, N.; Valeriano, J.; Granados, E.

2015. Aislamiento e identificación de Phytophthora cinnamomi Rands en el cultivo de palto variedades Hass y Fuerte. CenciAgro, 1: 57-63.

CENAGRO.

1994. III Censo Nacional Agropecuario 1994. Censo Nacional Agropecuario. INEI. Lima, Perú. 18 p.
CENAGRO.

2012. IV Censo Nacional Agropecuario 2012. Censo Nacional Agropecuario. INEI. Lima, Perú. 63 p.

Malagon R.; Praguer, M.

2001. El enfoque de sistemas: Una opción para el análisis de las unidades de producción agrícola. Universidad Nacional de Colombia. Palmira. Colombia. 190 p.

Márquez, F.; Julca, A.; Canto, M.; Soplín, H.; Vargas, W.; Huerta, P.

2016. Sustentabilidad ambiental en fincas cafetaleras después de un proceso de certificación orgánica en la convención (Cusco, Perú). Ecología Aplicada, 15(2): 125-132.

Minagri.

2002. Lineamientos de política agraria para el Perú. Proyecto PROAPA-GTZ. Ministerio de Agricultura y Riego Lima, Perú. 78 p.

Minagri.

2015. La palta producto estrella de exportación; tendencias de la producción y el comercio de la palta en el mercado internacional y nacional. MINAGRI - DGPA. Lima, Perú. 81 p.

Minagri.

2019. La situación del mercado internacional de la palta. MINAGRI - DGPA. Lima, Perú. 41 p.

Santistevan, M.; Julca, A.; Helfgott, S.

2015. Caracterización de las fincas productoras del cultivo de limón en las localidades de Manglaralto y Colonche, (Santa Elena, Ecuador). Revista Científica y Tecnológica UPSE, 3(1): 133-142.

Santistevan, M.; Julca, A.; Borjas, R.; Tuesta, O.

2014. Caracterización de fincas cafetaleras en la localidad de Jipijapa (Manabí, Ecuador). Ecología Aplicada, 13(2): 187-192.

Servicio Nacional de Meteorología e Hidrología (Senamhi).

2017. Senamhi (Tacna y Moquegua). Disponible en: www. tacna.senamhi.gob.pe/. Consultado: 1/abr/ 2017.

Sheaffer, R.; Mendenhall, W.; Ott, L.

1987. Elementos de muestreo. Grupo Editorial Iberoamericana. México. $321 \mathrm{p}$.

Tuesta, O.; Julca, A.; Borjas, R.; Rodríguez, P.; Santistevan, M. 2014. Tipología de fincas cacaoteras en la subcuenca media del río Huayabamba, distrito de Huicungo (San Martín, Perú). Ecología Aplicada, 13(2): 71-78. 
